\title{
Clinical Document Architecture
}

National Cancer Institute

\section{Source}

National Cancer Institute. Clinical Document Architecture. NCI Thesaurus. Code C142426.

A Health Level 7 specification containing the structure and semantics that clinical documents should follow in order to facilitate data exchange. 